\title{
On-Orbit Lunar Modulation Transfer Function Measurements for the Moderate Resolution Imaging Spectroradiometer
}

\author{
Taeyoung Choi, Xiaoxiong Xiong, and Zhipeng Wang
}

\begin{abstract}
Spatial quality of an imaging sensor can be estimated by evaluating its modulation transfer function (MTF) from many different sources such as a sharp edge, a pulse target, or bar patterns with different spatial frequencies. These well-defined targets are frequently used for prelaunch laboratory tests, providing very reliable and accurate MTF measurements. A laboratory-quality edge input source was included in the spatial-mode operation of the Spectroradiometric Calibration Assembly (SRCA), which is one of the onboard calibrators of the Moderate Resolution Imaging Spectroradiometer (MODIS). Since not all imaging satellites have such an instrument, SRCA MTF estimations can be used as a reference for an on-orbit lunar MTF algorithm and results. In this paper, the prelaunch spatial quality characterization process from the Integrated Alignment Collimator and SRCA is briefly discussed. Based on prelaunch MTF calibration using the SRCA, a lunar MTF algorithm is developed and applied to the lifetime on-orbit Terra and Aqua MODIS lunar collections. In each lunar collection, multiple scan-direction Moon-to-background transition profiles are aligned by the subpixel edge locations from a parametric Fermi function fit. Corresponding accumulated edge profiles are filtered and interpolated to obtain the edge spread function (ESF). The MTF is calculated by applying a Fourier transformation on the line spread function through a simple differentiation of the ESF. The lifetime lunar MTF results are analyzed and filtered by a relationship with the Sun-Earth-MODIS angle. Finally, the filtered lunar MTF values are compared to the SRCA MTF results. This comparison provides the level of accuracy for on-orbit MTF estimations validated through prelaunch SRCA measurements. The lunar MTF values had larger uncertainty than the SRCA MTF results; however, the ratio mean of lunar MTF fit and SRCA MTF values is within $2 \%$ in the $250-$ and $500-\mathrm{m}$ bands. Based on the MTF measurement uncertainty range, the suggested lunar MTF algorithm can be applied to any on-orbit imaging sensor with lunar calibration capability.
\end{abstract}

Index Terms-Aqua, MODIS, moon, MTF, spatial quality, SRCA, Terra.

Manuscript received March 28, 2012; revised July 31, 2012, and December 3, 2012; accepted December 28, 2012.

T. Choi is with Sigma Space Corporation, Lanham, MD 20706 USA, and also with George Mason University, Fairfax, VA 22030, USA (e-mail: taeyoung.choi@sigmaspace.com).

X. Xiong is with the Goddard Space Flight Center, National Aeronautics and Space Administration, Greenbelt, MD 20771 USA (e-mail: Xiaoxiong. Xiong-1@nasa.gov).

Z. Wang is with Sigma Space Corporation, Lanham, MD 20706 USA (e-mail: zwang@sigmaspace.com).

Color versions of one or more of the figures in this paper are available online at http://ieeexplore.ieee.org.

Digital Object Identifier 10.1109/TGRS.2013.2238545

\section{INTRODUCTION}

\section{A. MTF as a Measure of Spatial Quality}

In the era of an ever-increasing number of Earth-observing imaging sensors, spatial quality is one of the most important sensor characteristics, along with radiometric and geometric performance. As a standard measure of this spatial quality, the modulation transfer function (MTF), particularly its value at the Nyquist frequency, has been utilized in sensor design and product specifications [1], [2]. The MTF is a normalized magnitude response of the optical transfer function in the frequency domain. It is obtained through a Fourier transformation of a point spread function (PSF) in the spatial domain. The PSF is influenced by the system's optical and detector components, as well as sensor motion and electronic effects. The system PSF is defined as a 2-D spatial convolution of its subcomponents. There were previous attempts to measure the 2-D PSF using an array of black squares on a white sand surface [3] and using convex mirrors [4], but the 2-D responses were too noisy to fit a surface Gaussian function properly when recording ground collections from space. This technique has been improved in recent research using a grid of spherical reflectors providing PSF changes over time [5]. Instead of using the 2-D PSF, various techniques have been developed to estimate a 1-D PSF, known as a line spread function (LSF), using a source sharp edge, a pulse, and patterned targets [6]-[8].

Aside from directly measuring the PSF and LSF of a system, the functions can be calculated after imaging a target. A sharp straight-edged target is most frequently used and measured at a slight angle from the image vertical direction, to provide subpixel-level resolution. For high-spatial-resolution sensors such as IKONOS and QuickBird, tarps are carefully deployed at locations on the Earth's surface to construct a ground edge target [2]. After applying an edge detection algorithm and proper filtering of the aligned edge profiles, an edge spread function (ESF) is derived. There are different parametric and nonparametric fitting techniques used throughout the process to properly calculate an accurate MTF, such as Gaussian function fit, sigmoid function fit, modified Savitzky-Golay (SG) filtering, and LOcally WEighted Scatterplot Smoothing fit [2], [4], [6], [7]. The derivative of the ESF is the LSF. Once the LSF is found, a normalized Fourier transformation is applied to get the imaging system's MTF.

Alternatively, a pulse method is applied when the spatial resolution of the testing sensor is too large to construct a large and 
uniform edge target. The pulsewidth is chosen to be 1.5 times the ground sample distance (GSD) to have the desired signal strength to avoid the first zero location of the "sinc" function occurring around the Nyquist frequency [4]. For the Landsat 7 Enhanced Thematic Mapper Plus MTF evaluation, a doublespan bridge over the Lake Pontchartrain Causeway in Louisiana served as a pulse target for a panchromatic band with $10-\mathrm{m}$ GSD and multispectral bands with 30-m GSD [8]. Performance changes are detected by comparing on-orbit MTF to prelaunch modeling of the optical system.

\section{B. Sensor Overview}

The first Moderate Resolution Imaging Spectroradiometer (MODIS) is on board the Terra spacecraft and was launched on December 18, 1999, whereas the second MODIS is on board the Aqua spacecraft and was launched on May 4, 2002. These two instruments are nearly identical in design and specifications. MODIS is designed to extend heritage sensors' data records with improved capabilities in spatial, spectral, and temporal measurements for the National Aeronautics and Space Administration's Earth Observing Systems [9], [10]. Each MODIS has 36 observation bands with spatial resolutions at nadir of $250 \mathrm{~m}$ for bands 1 and 2, $500 \mathrm{~m}$ for bands $3-7$, and $1 \mathrm{~km}$ for bands $8-36$. The $250-\mathrm{m}, 500-\mathrm{m}$, and $1-\mathrm{km}-$ resolution bands each have 40, 20, and 10 detectors, respectively, in the along-track direction, with a total of 490 individual detectors. There are 20 reflective solar bands (RSBs) with spectral wavelength coverage of $0.41-2.2 \mu \mathrm{m}$ and 16 thermal emissive bands (TEBs) with spectral wavelength coverage of 3.75-14.4 $\mu \mathrm{m}$. The key design specifications and calibration requirements are well documented by Xiong and Barnes [10]. The MODIS onboard calibrators include a solar diffuser (SD), an SD stability monitor (SDSM), a Spectroradiometric Calibration Assembly (SRCA), a blackbody (BB), and a space view (SV) port. The SD and SDSM are used for RSB calibrations, whereas the BB is used for TEB calibrations [11], [12]. MODIS collects data using a double-sided scan mirror over a large scan angle range. The onboard calibrators are located opposite the Earth view port, so that one side of the mirror views calibrators when the other side views the Earth continuously as the scan mirror rotates.

\section{MODIS SPATIAL CHARACTERIZATION FROM PRELAUNCH TO ON-ORBIT}

\section{A. Prelaunch MTF Characterization from IAC}

The MODIS prelaunch spatial characterization was performed by a ground calibration source called the Integrated Alignment Collimator (IAC), which uniformly filled the full MODIS aperture [13]. The IAC has two calibration sources, i.e., the $\mathrm{BB}$ and the integration sphere to illuminate all the RSBs and TEBs. The input beam is collimated after passing through a chopper, a reticle, the track-axis fold mirror, and the scan-axis fold mirror. These scan- and track-axis mirrors are aligned with the testing sensor's imaging axis. The position and motion of the slit are controlled by two laser interferometers. The collimated beam is then directed to MODIS, covering the

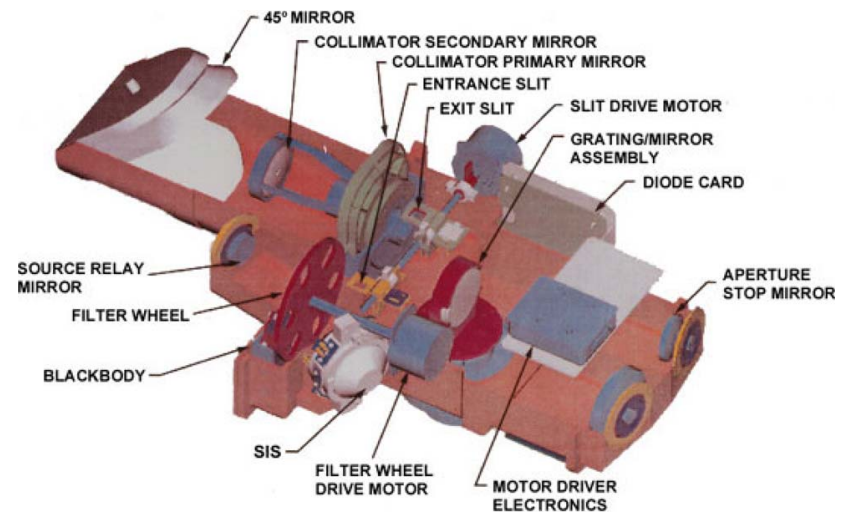

Fig. 1. SRCA [13].

entire pupil of the sensor. The slit covers the entire detector with a width of one-tenth of the detector size. To enhance the LSF measurement accuracy, a finer phase delay is used with higher resolution bands. For 250- and 500-m bands, a resolution equal to 0.1 times the instantaneous field of view (IFOV) is used, whereas 0.2 IFOV was applied to $1-\mathrm{km}$ bands. The slit size is narrow enough so that the slit responses can be accepted as impulse responses in the perpendicular direction of the pulse. Although IAC measurements are finely sampled, a uniformly sampled LSF is recalculated through a cubic spline interpolation. Finally, the Fourier transform is applied and normalized to obtain the MTF.

\section{B. Prelaunch MTF Characterization from SRCA}

During the prelaunch tests, SRCA spatial-mode collections were performed along with the IAC measurements simultaneously under the same ambient temperature condition. As shown in Fig. 1, a spherical integration source (SIS) and an infrared source are deployed to cover a wide spectral range. Within the SIS, there are four $10-\mathrm{W}$ and two $1-\mathrm{W}$ lamps providing various illumination levels. The infrared source, which is a heat resistance source, can be powered to a temperature range between $385 \mathrm{~K}$ and $395 \mathrm{~K}$ for the thermal band calibration [14]. The SIS and infrared sources are combined through a dichroic beam combiner at a specific filter wheel selection to cover all the MODIS spectral bands. The light transmits through a rectangular reticle and becomes a rectangle pattern of ground equivalent $5 \mathrm{~km}$ by $12 \mathrm{~km}$ in scan and track directions. This rectangular pattern provides a uniform response with high signal-to-noise sharp edges on the long sides of the rectangle. In SRCA spatial mode, the monochromator is replaced by a relay collimator. Sensor responses to a patterned light source with a desired illumination level, or SNR, are collected with a set of phase delays which provide pulsewidth profiles in 0.2-IFOV subsample resolutions. For each band/detector, an accumulated pulse response (APR) is constructed from all the pulse profiles with five independent phase delays [14], [15]. A cubic spline interpolation is applied to get a uniformly sampled APR at 0.05 subpixel resolution. The two edge profiles from the APR are differentiated to get the corresponding LSF. Finally, the SRCA MTF is calculated from the LSF by applying the Fourier transformation. 


\section{On-Orbit MTF Characterization from SRCA}

The SRCA has spatial, spectral, and radiometric operational modes. The spatial mode was operated every other month early in the mission and changed frequency to quarter-annual operation after a second lamp failure for both Terra and Aqua. The spatial-mode SRCA collections were utilized to track changes of the imaging system's MTF and band-to-band registration over time in all 36 bands [16]. Since the SRCA spatial modes are operated in exactly the same way in prelaunch and on-orbit collections, the collections are processed as described in the previous section. A unique advantage of SRCA spatial-mode collection is superior signal strength to the noise level. SNR was calculated from the height of the edge divided by the noise level from both sides of the uniform areas. The average standard deviation (STD) values for either side of the edges from the APR indicated [13] that the calculated SNR is approximately four times higher than the desired SNR value (100) for reliable MTF estimations [17]. Consequently, SRCA spatial-mode operations have provided very stable and reliable sharp edge profiles free of atmospheric effects for accurate measurements of the MODIS imaging system MTF.

Because the SRCA aperture only covers about one-fifth of the MODIS aperture, the SRCA MTF measurement may introduce small biases due to the smaller coverage of full optical path. On the other hand, the prelaunch testing results have provided accurate MODIS MTF measurements from IAC with a full aperture optical system. As a result, the IAC MTF serves as a reference because of its high-quality optics and full aperture coverage compared to the partial coverage of SRCA. A set of SRCA correction factors is derived from measurements made at the same time with the IAC to mitigate the MTF differences and transfer the ground-based IAC reference to the SRCA [14], [15]. Through these steps, the IAC successfully transferred prelaunch spatial calibration accuracy to on-orbit SRCA spatial calibrations.

\section{ON-ORBIT MODIS LUNAR MTF}

\section{A. General Information on MODIS Lunar Collections}

A lunar image collection is obtained through the MODIS SV port. The Moon is considered a stable radiometric reference for the visible and near-infrared spectrum. However, its irradiance is strongly dependent on viewing geometry. Only after correcting for the geometric effects can the lunar irradiance be used to monitor a sensor's long-term radiometric stability [18]. For radiometric calibration purposes, lunar observations have been made regularly with a phase angle range from $55^{\circ}$ to $56^{\circ}$ for Terra MODIS and from $-56^{\circ}$ to $-55^{\circ}$ for Aqua MODIS. In general, both instruments can acquire Moon observations approximately eight or nine times per year. For each lunar collection, MODIS views the Moon with multiple scans per observation. From these multiple lunar observations, sharp edge profiles can be found on the right side of the Moon and aligned to construct normalized accumulated edge profiles. However, the Moon images have a clear circular edge on the right and blurry shadow lines on the left, as shown in Fig. 2. At the start of a lunar collection, the Moon appears from the bottom of

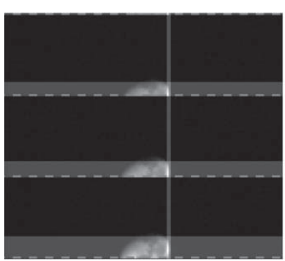

(a)

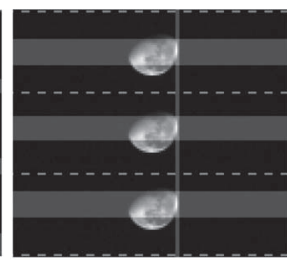

(b)

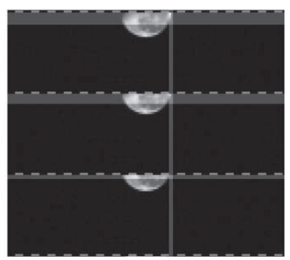

(c)
Fig. 2. Terra lunar collection images on March 24, 2000, in band 1. The figure shows three consecutive scans, and the dashed lines indicate the first detector out of 40 detectors in a scan. The highlighted vertical line denotes the two columns of the right-side Moon edge. The selected rows are shown as highlighted horizontal lines. (a) Start of lunar collection. (b) Middle of lunar collection. (c) End of lunar collection.

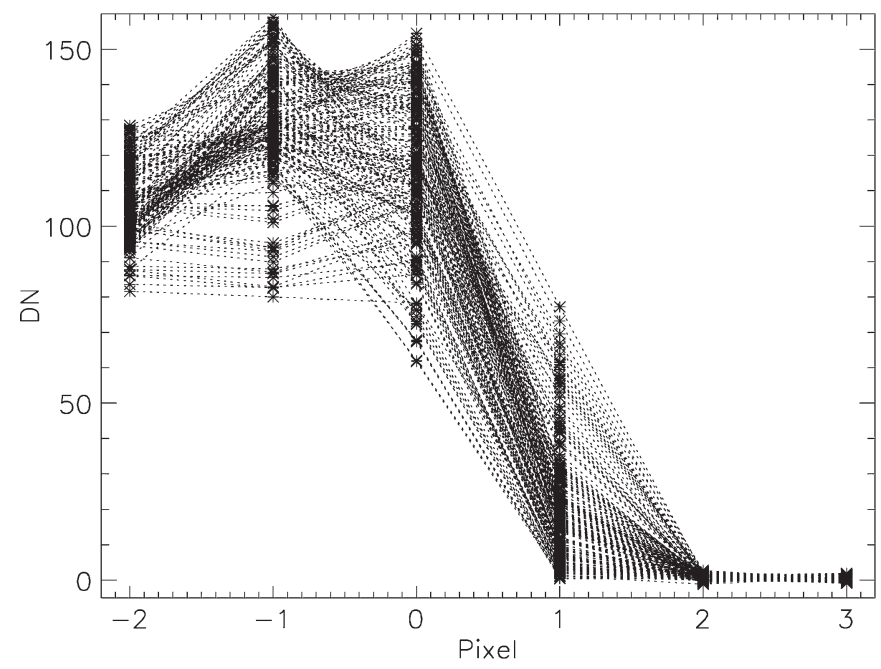

Fig. 3. All the selected edge profiles around the right edge of the Moon on March 24, 2000, in band 1 .

the scan line, as shown in Fig. 2(a). The scan lines cover the whole visible Moon in the middle of lunar collection, and the Moon gradually disappears at the end of the Moon collection, as shown in Fig. 2(b) and (c). These multiple scan lines of observations are combined to construct an edge transition profile near the right side of the Moon.

\section{B. Lunar MTF Algorithm Description}

The diameter of the Moon image on the focal plane is approximately 28 pixels for the $250-\mathrm{m}$ band 1 in scan and track directions, as shown in Fig. 2. In both Terra and Aqua lunar collections, the sharp vertical edges appear at the right side of the Moon, whereas the left edges are affected by a dark shadow due to the relative positions of the Sun, Moon, and MODIS. Considering lunar collection conditions such as phase angles and direction of shade, the two rightmost pixel columns of the Moon image are selected in the along-scan direction, which contains greater than 5\% of the maximum Moon illumination digital number (DN). These selected rows, based on the rightmost Moon pixels, are highlighted in the multiple horizontal scans of the Moon in Fig. 2. Partial Moon images can be seen in multiple scans toward the start and end of a collection. All the horizontal profiles from the right side of the Moon edges are utilized for MTF characterization. Fig. 3 shows all the selected profiles of the right side of the Moon plotted without 


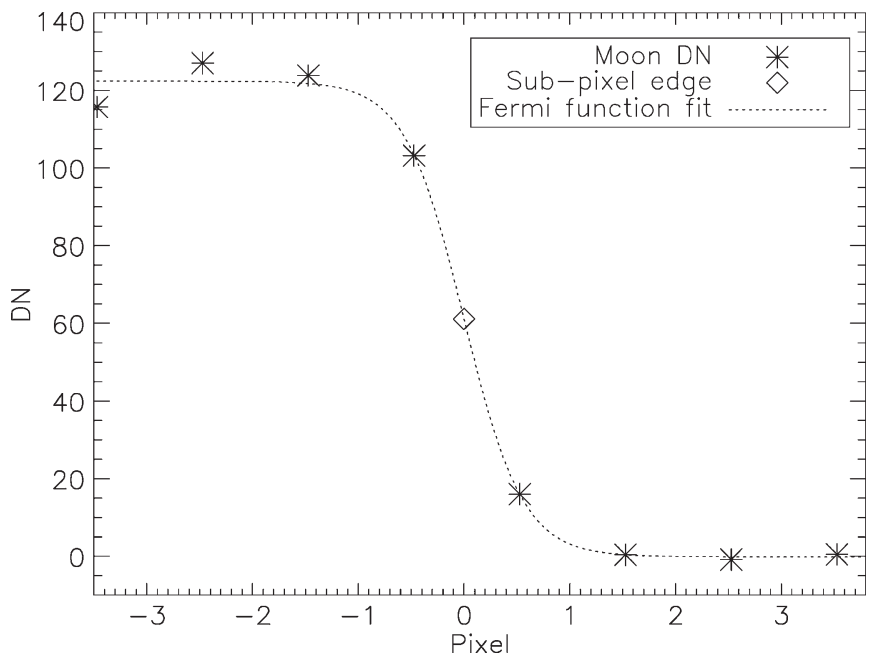

Fig. 4. Subpixel edge location was found by fitting the Fermi function to the Moon profile.

edge alignment. The DN level of dark space is very stable and near zero after background subtraction, but the Moon-side DN level ranges from approximately 80 to $160 \mathrm{DN}$. Because most of the rows have different levels of transition from the dark space to the nonuniform Moon surface, the selected rows need to be normalized before they are accumulated according to their subpixel edge locations. For each row profile in the scan direction, an initial maximum DN transition point or a rough estimate of the Moon edge location is determined by differentiation filtering. A nonuniformity test and normalization are then applied to these near Moon edge pixels in each edge profile. The absolute DN value differences in the first and second pixels (locations -2 and -1 in Fig. 3) are compared to the edge transition level. The valid rows are selected when the DN difference is less than $10 \%$ of the edge height. After the Moon side stability filtering, a subpixel edge location is found in each row profile, as shown in Fig. 4. In the figure, the pixel-based Moon ESF is marked with asterisks, and the corresponding subpixel edge location is evaluated by fitting the ESF to a Fermi function [19]

$$
f(x)=\frac{a}{e^{\left[\frac{(x-b)}{c}+1\right]}}+d .
$$

In the aforementioned Fermi function equation, the parameter $a$ is a scale factor, $b$ is the curve inflection point, $c$ represents edge sharpness, and $d$ is a bias level. After finding the best fit parameters, the $b$ value is recognized as the subpixel edge location of the edge profile. Then, the $x$ positions of the ESF profiles are shifted in reference to the subpixel edge location, shown as the diamond symbol in Fig. 4. The ESF is normalized by the fitted Fermi function at pixel location -3 , which is a good approximation of the edge height scaling factor. The subpixel edge detection and ESF profile normalization are applied to all the selected rows and aligned by a common subpixel edge location, seen as the origin along the $x$-axis in Fig. 5. After the edge profiles are normalized and aligned, an accumulated edge profile is still not ready for the Fourier transformation, mainly because the profile is no longer uniformly sampled. In addition, variations of the Moon-edge illumination need to be

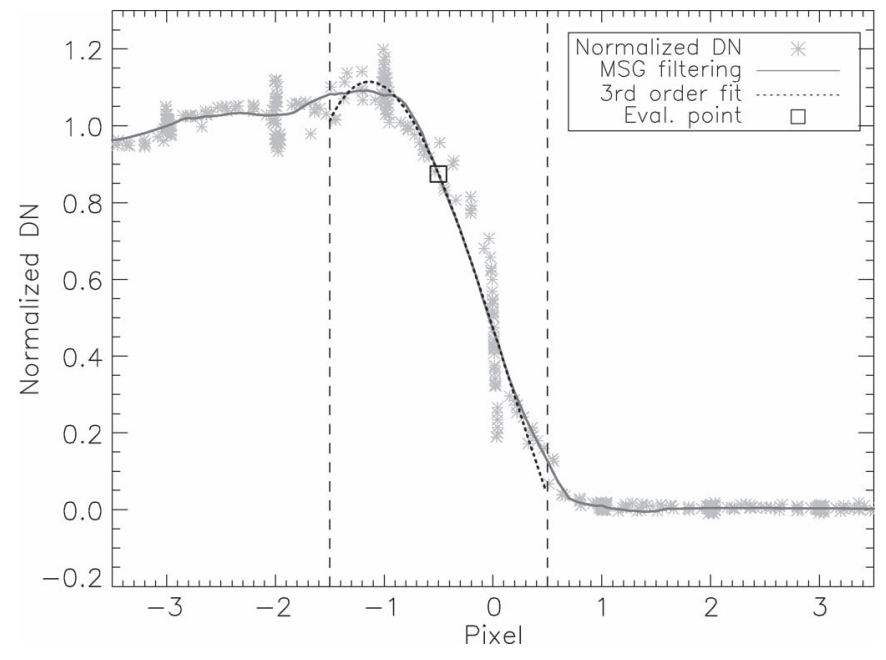

Fig. 5. Accumulated edge profile after normalization and edge alignment from Terra lunar collection on March 24, 2000, in band 1. MSG filtering profile is shown as a solid line within the two-pixel window from -1.5 to 0.5 as an example. A third-order polynomial was fitted from the profile. The accumulated edge profile data points are clustered near the discrete pixel locations due to the repeating lunar edges at the similar subpixel edge locations with the MODIS sampling.

filtered. A modified version of the SG filter is applied to get a uniformly sampled smooth profile, as shown as the solid line in Fig. 5 [17]. The modified SG filtering is a sliding window filter with a fixed window size. In this application, a twopixel-wide window and a third-order polynomial fitting are applied to the noisy nonuniformly sampled data points within the window. In the middle of the defined window, the final filtered value is evaluated from the fitted polynomial. The thirdorder polynomial fit and an evaluation point are shown in Fig. 5 as a dotted line and a square symbol, respectively, within the example window from -1.5 to 0.5 pixel locations. The sliding step of the window is $1 / 20$ of a pixel, which determines the resolution of the modified SG filter output resolution. A simple differentiation filtering and normalization are applied on the ESF to calculate the LSF shown in Fig. 6. The LSF profile is noisier than the ESF because of the characteristics of the differential filtering. The LSF range extends to two pixels on either side of the maximum point because the edge transition in the ESF is stabilized sufficiently within four pixels. Finally, the MTF is calculated by applying a Fourier transformation to the LSF. The frequency axis is normalized to the Nyquist frequency, and corresponding MTF values are shown in Fig. 7 at $0.25,0.5,0.75$, and 1 Nyquist frequency, as in the MODIS specification [1].

\section{RESUlts AND Discussions}

Based on the algorithm detailed in the previous section, Terra on-orbit lunar MTF values are calculated using lifetime lunar collections, as shown in Fig. 8. The numbers indicate years from 2000, and the dotted lines are year division points. The diamond symbols are average SRCA MTF values, and the asterisks represent Terra lunar MTF values. The SRCA MTF values are more consistent and stable than the lunar MTF. This is expected due to advantages of the SRCA, including a straight edge 


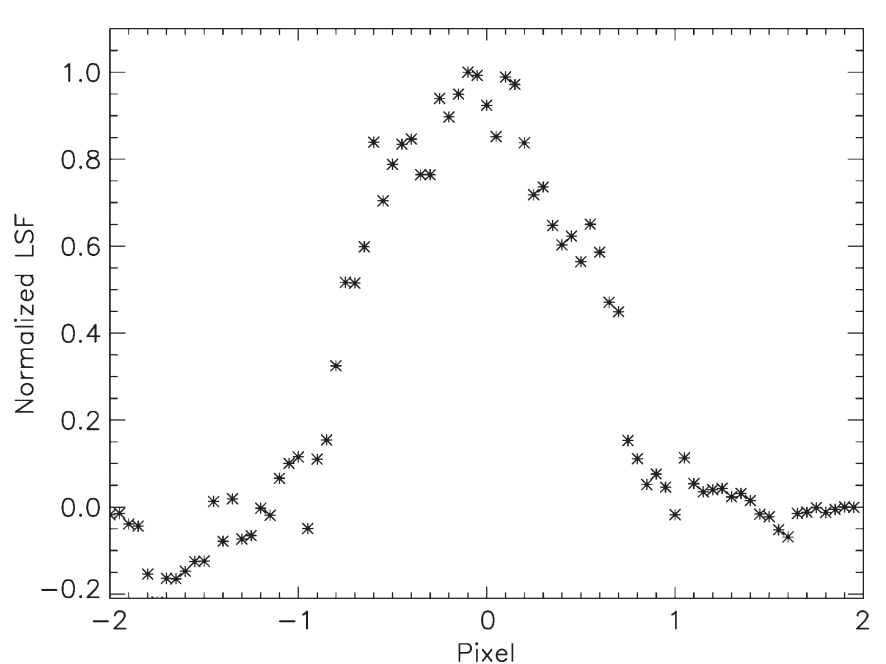

Fig. 6. LSF profile was calculated from a simple differentiation filtering on ESF for Terra lunar collection on March 24, 2000, in band 1.

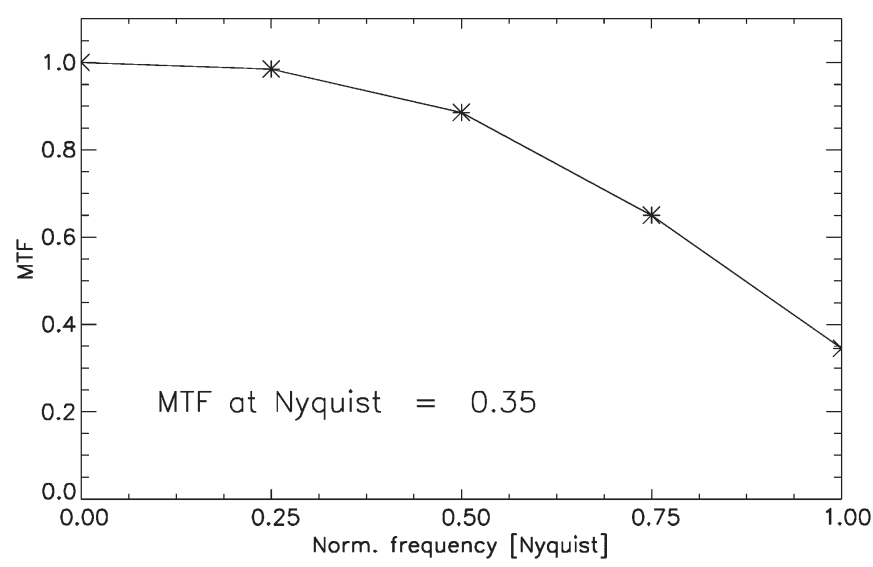

Fig. 7. MTF for Terra lunar collection on March 24, 2000, in band 1.

along the track direction, very high SNR between the bright and dark regions, and accurate and uniform sampling grids in nadir angle observations. An edge target with SNR values greater than 100 provides a particularly reliable MTF estimation through noise and PSF simulations [14], [17], which enables the SRCA results to be used as a reference for lunar MTF estimates. There are small but noticeable noises/oscillations in the lunar MTF results. Nevertheless, the multiyear lunar MTF results follow closely with the SRCA MTF values.

The impact due to system parameters on the lunar MTF has been tested and investigated to mitigate the noise. One of the geometric parameters from lunar collections, i.e., the Sun, Earth, and MODIS (SEM) angle, has a strong relationship with lunar MTF measurement accuracy. The SEM angles for each lunar observation range from approximately $130^{\circ}$ to $250^{\circ}$. When the SEM angle is near the maximum of its range, the dark nonuniform portion of the Moon is viewed on the right side of the image. Fig. 9(a) shows an example plot of the dark portion of the Moon with a SEM angle of $243.7^{\circ}$. In this figure, nonuniform spatial patterns are observed near the right edge. These patterns cause ESF profiles to be noisy, and this noise propagates throughout the MTF calculation. When

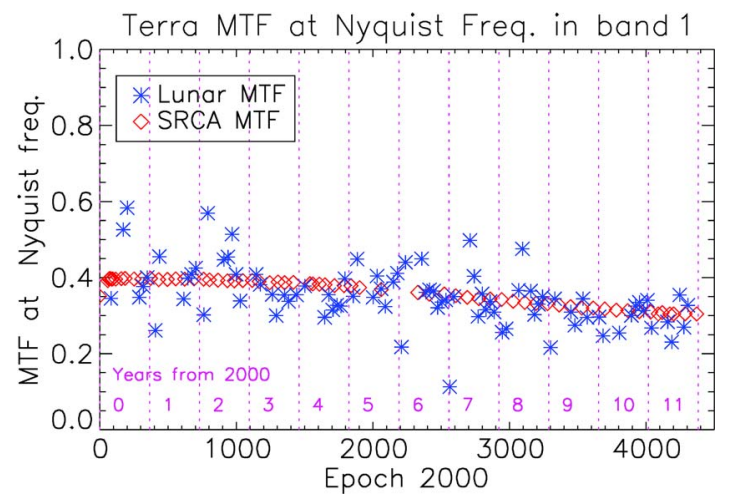

Fig. 8. Terra SRCA and lunar MTF values at Nyquist frequency in band 1 from 2000 to 2011. Numbers from 0 to 11 indicate years from 2000, and dotted lines are year division points.
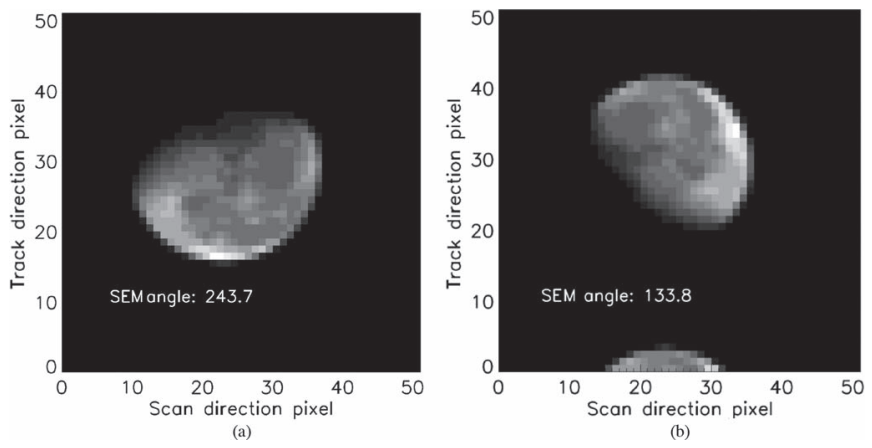

Fig. 9. Lunar surface plots at different Sun, Earth, and MODIS (SEM) angles (Terra MODIS). (a) Lunar collection on January 23, 2011. (b) Lunar collection on September 17, 2011.

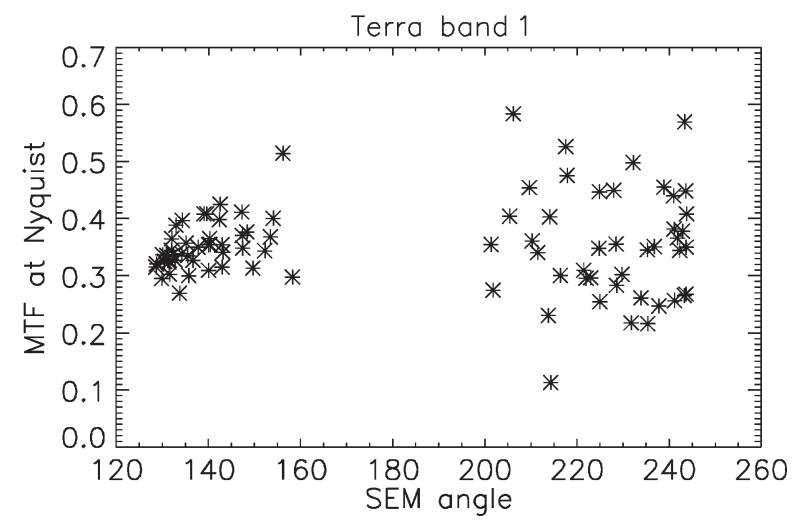

Fig. 10. Terra SRCA and lunar MTF values at Nyquist frequency in band 1 from 2000 to 2011.

the SEM angle is near the minimum of its range, the bright and uniform side of the Moon is observed on the right side of the image. Fig. 9(b) shows the bright and uniform side of the Moon with a SEM angle of $133.8^{\circ}$. There is a significant relationship between SEM angles and lunar MTF values at Nyquist frequency, as shown in Fig. 10. When the SEM angle is less than $180^{\circ}$, Terra band-1 lunar MTF values are clustered tightly around 0.35 at Nyquist frequency. The lunar MTF values are noisier and more widespread for collections with SEM angles larger than $180^{\circ}$. The observed uniformity and illumination of the Moon are dependent on the SEM angle and 
This article has been accepted for inclusion in a future issue of this journal. Content is final as presented, with the exception of pagination.
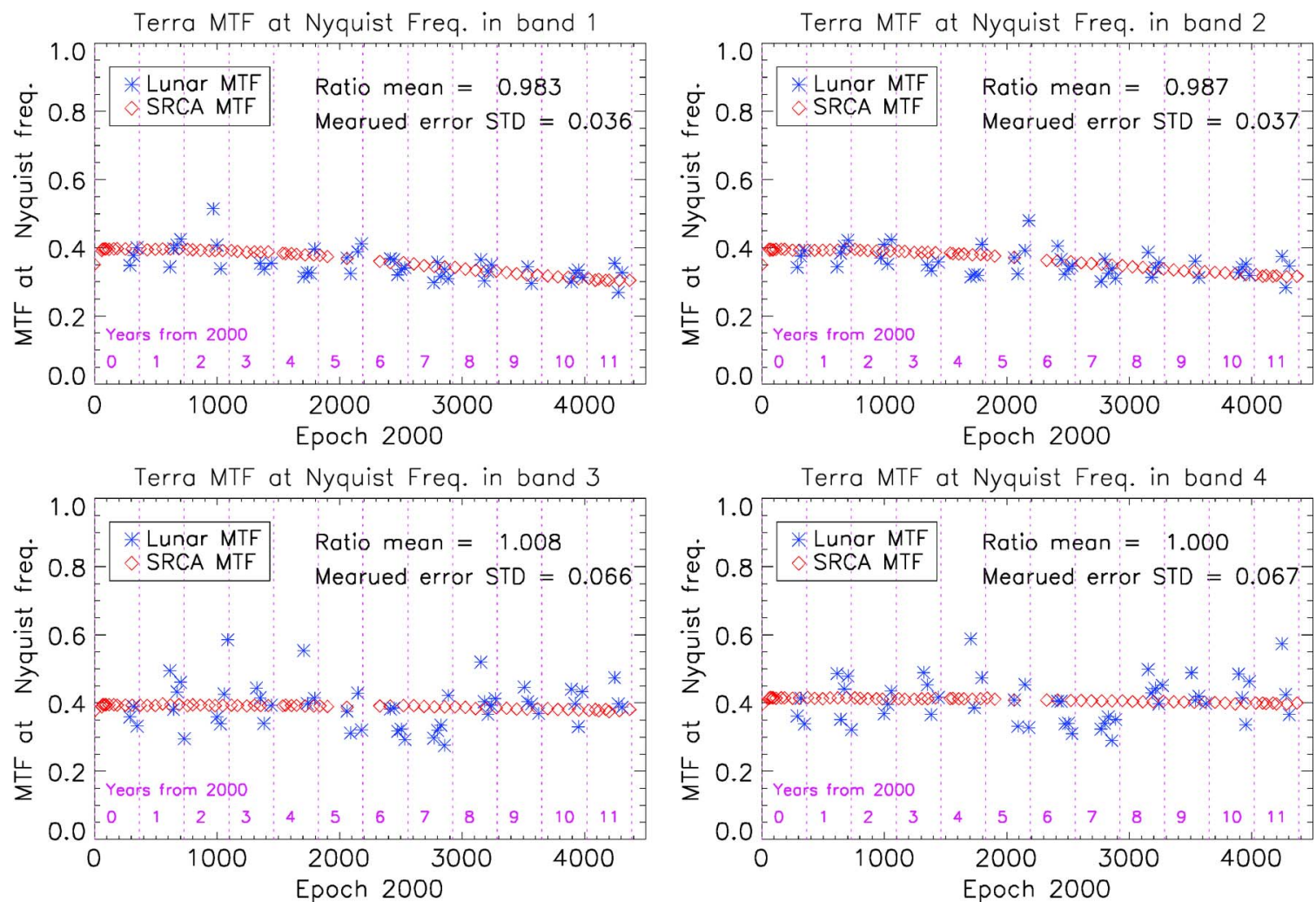

Fig. 11. Filtered Terra lunar MTF values at Nyquist frequency in bands 1-4. The "ratio mean" and "measured error STD" are based on the lunar MTF values with linear interpolated SRCA MTF values. The numbers from 0 to 11 indicate years from 2000, and dotted lines are year division points.

directly affect the quality of MTF estimates. With SEM angles greater than $180^{\circ}$, the area near the right edge of the observed Moon is dark and nonuniform. The Moon surface near the right edge is mostly bright and uniform with SEM angles less than $180^{\circ}$.

From these analyses, the SEM angle dependence is applied to lunar MTF results by filtering out MTF values with angles larger than $180^{\circ}$. Fig. 11 shows the filtered Terra lunar MTF values and their relation to SRCA MTF values in bands 1-4. This filtered set of MTF values seems to have reduced the noise seen earlier (in Fig. 8), and the trend is more consistent with that of the SRCA MTF values of band 1. At each lunar MTF measurement, a measured error is calculated by subtracting a lunar MTF value from a linearly interpolated SRCA MTF value. Similarly, the ratio mean is calculated by taking the ratios between lunar MTF values and SRCA MTF values interpolated at the corresponding lunar collection times, and then, all of the ratios are averaged. Corresponding ratio mean and STD values of all the measured errors are shown in Fig. 11. From the ratio mean, the lunar MTF value at Nyquist frequency is $1.7 \%$ less than the SRCA MTF value at Nyquist frequency in band 1 . The band-2 ratio mean and measured error STD values are very similar to those of band 1 . When this lunar MTF algorithm is applied to 500-m bands 3 and 4, the MTF results are nosier than the 250-m results with larger measured error STD values. This is expected since 500-m bands have coarser resolution than $250-\mathrm{m}$ bands and there are fewer rows and data points. It is apparent when a $500-\mathrm{m}$-band image is compared to a $250-\mathrm{m}$ simultaneous collection (Figs. 12 and 9(b), respectively). Along

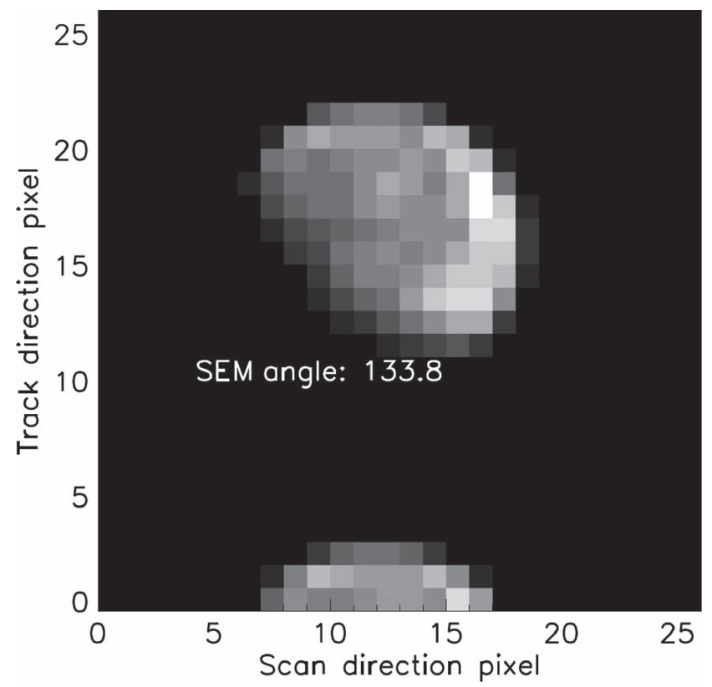

Fig. 12. Example of 500-m-resolution lunar image in band 3 on September 17, 2011 (Terra MODIS).

with this resolution issue, the ESF becomes easily affected by the noise with a small number of pixels and the size limit of uniform area on the Moon.

The ratio mean and measured error STD values are summarized in Table I. The first four MODIS bands show relations between lunar MTF and SRCA MTF within 2\% accuracy after the SEM angle filtering. Although the ratios and STDs are higher in the 500-m bands, the lunar MTF algorithm can still be successfully implemented to produce reasonable estimates 
TABLE I

Terra Lunar MTF Ratio and Measured ERror STD at NyQuist FreQuency AFTER SEM ANGLE FILTERING

\begin{tabular}{|c|c|c|c|c|}
\hline Band & 1 & 2 & 3 & 4 \\
\hline Resolution & $250 \mathrm{~m}$ & $250 \mathrm{~m}$ & $500 \mathrm{~m}$ & $500 \mathrm{~m}$ \\
\hline Ratio mean & 0.983 & 0.987 & 1.008 & 1.000 \\
\hline STD & 0.036 & 0.037 & 0.066 & 0.067 \\
\hline
\end{tabular}

TABLE II

Aqua LunAR MTF RATIO AND MEASURED ERRor STD AT Nyquist Frequency After SEM ANGLE FILTERING

\begin{tabular}{|c|c|c|c|c|}
\hline Band & 1 & 2 & 3 & 4 \\
\hline Resolution & $250 \mathrm{~m}$ & $250 \mathrm{~m}$ & $500 \mathrm{~m}$ & $500 \mathrm{~m}$ \\
\hline Ratio mean & 0.978 & 1.004 & 1.030 & 1.042 \\
\hline STD & 0.052 & 0.054 & 0.099 & 0.094 \\
\hline
\end{tabular}

when compared with SRCA MTF values. The proposed lunar MTF algorithm is not applicable for the MODIS 1-km-band lunar observations due to the limited number of available profiles in the region of interest. In addition, the lower resolution bands detect coarser profiles and lack the required fidelity needed to produce reliable MTF results.

For the Aqua lunar MTF, 45 collections were processed from 2002 to 2011. The same lunar MTF algorithm is successfully applied to the selected 250- and 500-m bands. The comparison between the Aqua lunar MTF results and the SRCA MTF values is summarized in Table II. The ratio mean of Aqua lunar MTF to SRCA MTF values at Nyquist frequency in the investigated bands and the measured error STD values are slightly larger than those of the Terra case; however, the Aqua lunar and SRCA MTF ratio mean values are all within $4 \%$ for both the Terra and Aqua sensors.

\section{CONCLUSION}

Spatial quality of MODIS is measured by the MTF, which is derived from the ESF and the LSF. The prelaunch Terra and Aqua MTF measurement by the IAC is transferred to the onorbit SRCA spatial-mode calibration, which serves as a fiducial MTF measurement. As an independent source of ESF, a lunar MTF algorithm is developed and applied to the Terra and Aqua 250- and 500-m-resolution bands. Over the lifetimes of Terra and Aqua on-orbit operations, lunar MTF results at Nyquist frequency are well matched to the SRCA MTF measurements after a specific noise filtering process. The lunar MTF measurements have relatively large uncertainties because of lower SNR values in accumulated ESF profiles; however, a tight relationship is found by selecting a SEM angle less than $180^{\circ}$. The angle dependence, which describes the relative orientations among the Sun, Earth, and the spacecraft, affects the observed lunar surface uniformity and spatial pattern around the right edge of the Moon. The MTF noise level is reduced effectively by selecting and filtering MTF results to within the ideal SEM angle range. The ratio mean values between lunar MTF and interpolated SRCA MTF values at Nyquist frequency show less than $4 \%$ differences in the tested bands $1-4$ for both Terra and Aqua sensors.

This algorithm can be applied to check long-term spatial quality performance of sensors with lunar calibration ability from a proper combination of roll, yaw, and pitch maneuvers. To get reliable results, the characteristics of lunar collection of the tested sensor must be studied carefully to find the relationships between measured MTF and spatial noise or patterns on the Moon surface before making conclusions.

Future work includes performing this analysis on observations taken in the instrument track direction. Since there are no vertical MTF results from the SRCA, lunar MTF results in the vertical direction could provide a useful estimate of long-term changes and form a complete set of instrument scan- and trackdirection PSF or MTF.

\section{REFERENCES}

[1] R. Weber, Specification for the Moderate-Resolution Imaging Spectroradiometer (MODIS), 1993, Goddard Space Flight Center 422-20-02.

[2] R. Ryan, B. Baldridge, R. A. Schowengerdt, T. Choi, D. L. Helder, and S. Blonski, "IKONOS spatial resolution and image interpretability characterization," Remote Sens. Environ., vol. 88, no. 1/2, pp. 37-52, Nov. 2003.

[3] R. F. Rauchmiller and R. A. Schowengerdt, "Measurement of the Landsat Thematic Mapper MTF using an array of point sources," Opt. Eng., vol. 27, no. 4, pp. 334-343, Apr. 1988.

[4] D. L. Helder, T. Choi, and M. Rangaswamy, "In-flight characterization of spatial quality using point spread functions," in Proc. Int. Workshop Radiometric Geometric Calibration-In Post-Launch Calibration Satellite Sensors, 2004, pp. 151-170.

[5] S. Schiller, J. Silny, and M. Taylor, "In-flight performance assessment of imaging systems using the specular array radiometric calibration (SPARC) method," in Proc. Joint Agency Commercial Imagery Eval. Workshop, Fairfax, VA, USA, 2012, pp. 1-26.

[6] V. Kaftandjian, Y. Zhu, G. Roziere, G. Peix, and D. Babot, "A comparison of the ball, wire, edge, and bar/space pattern techniques for modulation transfer function measurements of linear x-ray detectors," J. X-Ray Sci. Technol., vol. 6, no. 2, pp. 205-221, Jan. 1996.

[7] K. Kohm, "Modulation transfer function measurement method and results for the Orbview-3 high resolution imaging satellite," in Proc. Geo-Imagery Bridging Continents XXth ISPRS Congr., 2004, pp. 7-12.

[8] J. Storey, "Landsat 7 on-orbit modulation transfer function estimation," in Proc. SPIE, Sens., Syst., Next-Gener. Satellites, 2001, pp. 50-61.

[9] W. L. Barnes, X. Xiong, and V. V. Salomonson, "Status of Terra MODIS and Aqua MODIS," Adv. Space Res., vol. 32, no. 11, pp. 2099-2106, Dec. 2003.

[10] Xiong, X. and W. L. Barnes, "An overview of MODIS radiometric calibration and characterization," Adv. Atmos. Sci., vol. 23, no. 1, pp. 69-79, Jan. 2006.

[11] X. Xiong, J. Sun, X. Xie, W. Barnes, and V. Salomonson, "On-orbit calibration and performance of Aqua MODIS reflective solar bands," IEEE Trans. Geosci. Remote Sens., vol. 48, no. 1, pp. 535-546, Jan. 2010.

[12] X. Xiong, B. N. Wenny, A. Wu, W. Barnes, and V. V. Salomonson, "Aqua MODIS thermal emissive bands on-orbit calibration, characterization, and performance," IEEE Trans. Geosci. Remote Sens., vol. 47, no. 3, pp. 803-814, Mar. 2009.

[13] [Online]. Available: http://modis.gsfc.nasa.gov/about/srca.php

[14] T. Choi, N. Che, and X. Xiong, "On-orbit Aqua MODIS modulation transfer function trending in along-scan from the Spectro-Radiometric Calibration Assembly," in Proc. SPIE, 2008, vol. 7081, pp. 70810A-170810A-10.

[15] T. Choi, N. Che, and X. Xiong, "On-orbit Terra MODIS modulation transfer function trending along-scan from the Spectro-Radiometric Calibration Assembly," in CALCON, Logan, UT, USA, 2007.

[16] X. Xiong, N. Che, and W. Barns, "Terra MODIS on-orbit spatial characterization and performance," IEEE Trans. Geosci. Remote Sens., vol. 43, no. 2, pp. 355-365, Feb. 2005.

[17] T. Choi and D. Helder, "Generic sensor modeling for modulation transfer function (MTF) estimation," in Proc. Pecora 16 'Global Priorities Land Remote Sens.', 2005, pp. 1-12. 
[18] J. Sun, X. Xiong, W. Barnes, and B. Guenther, "MODIS reflective solar bands on-orbit lunar calibration," IEEE Trans. Geosci. Remote Sens., vol. 45, no. 7, pp. 2383-2393, Jul. 2007.

[19] A. P. Tzannes and J. M. Mooney, "Measurement of the modulation transfer function of infrared cameras," Opt. Eng., vol. 34, no. 6, pp. 1808-1817, Jun. 1995

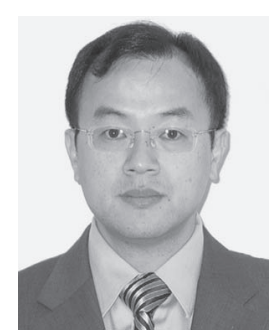

Taeyoung (Jason) Choi received the B.S. degree in electronics from Konkuk University, Seoul, Korea, and the M.S. degree in electrical engineering from South Dakota State University, Brookings, SD, USA. $\mathrm{He}$ is currently working toward the Ph.D. degree in earth systems and geoinformation sciences at George Mason University, Fairfax, VA, USA.

$\mathrm{He}$ is also currently with Sigma Space Corporation, Lanham, MD, USA, where he is a member of the Moderate Resolution Imaging Spectroradiometer (MODIS) Calibration Support Team performing radiometric, spectral, and spatial calibrations in reflective solar bands. Since 1999, he has been working in remote sensing, instrument calibration, and data applications with various sensors such as IKONOS, QuickBird, Thematic Mapper, Enhanced Thematic Mapper Plus, Advanced Land Imager, Hyperion, and MODIS.

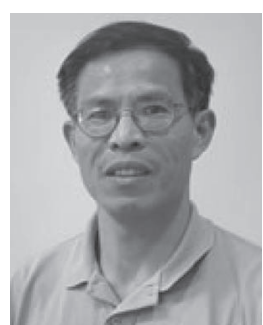

Xiaoxiong (Jack) Xiong received the B.S. degree in optical engineering from the Beijing Institute of Technology, Beijing, China, and the Ph.D. degree in physics from the University of Maryland, College Park, MD, USA.

He is currently an Optical Physicist with the Goddard Space Flight Center (GSFC), National Aeronautics and Space Administration (NASA), Greenbelt, MD, USA, where he is also the Moderate Resolution Imaging Spectroradiometer (MODIS) Project Scientist and the Technical Lead for both the MODIS Characterization Support Team and the Visible Infrared Imaging Radiometer Suite (VIIRS) Calibration Support Team. Before joining the GSFC, NASA, he had also worked in the fields of optical instrumentation, nonlinear optics, laser and atomic spectroscopy, and resonance ionization mass spectrometry at universities and industry and at the National Institute of Standards and Technology.

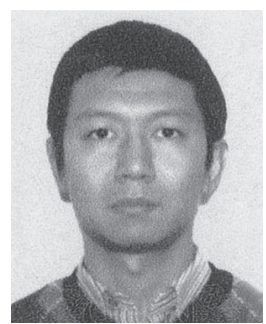

Zhipeng Wang received the B.S. and M.S. degrees in optoelectrics from the Department of Precision Instruments and Mechanology, Tsinghua University, Beijing, China, in 2000 and 2003, respectively, and the Ph.D. degree in optics from The University of Arizona, Tucson, AZ, USA, in 2008.

$\mathrm{He}$ is currently a Senior Support Scientist with Sigma Space Corporation, Lanham, MD, USA, working with the Moderate Resolution Imaging Spectroradiometer Characterization Support Team and the VIIRS Characterization Support Team. 